\section{Group performance on a multiple-solution task as a function of available time}

\author{
SURESH KANEKAR \\ Lawrence University, Appleton, Wis. 54911 \\ and \\ MILTON E. ROSENBAUM* \\ University of Iowa, Iowa City, Iowa 52240
}

The performance of four-member real groups on an anagrams task was compared to the output of nominal groups assembled from the response protocols of four individuals working alone. Three levels of time available for solution were employed. Production of words increased directly as a function of time for both types of groups, and nominal groups produced more words than real groups at each level of available time. There was no interaction between the two variables.

Several studies show that assembling groups to solve problems that permit multiple solutions provides no more, and often less, output than the amount that is obtained if a similar number of individuals work alone (cf. Shaw, 1971). Kelley \& Thibaut (1969) consider the possibility that the poorer performance by groups is based on time constraints that prevent groups from moving beyond the necessary but time-consuming activity of getting organized. They particularly cite a study by Anderson (1961) in which, as compared to a previous study by Watson (1928), twice as much time was permitted for an anagrams task.

Both Watson and Anderson compared the productivity of real groups with the output of nominal groups which were formed by pooling the response protocols of an appropriate number of individuals who had worked alone. Watson, who allowed $10 \mathrm{~min}$ for problem solving, found real groups inferior to nominal groups, while in the 20 min permitted by Anderson, the difference in performance between real and nominal groups was small and not significant. Anderson's data reveal that early in the time period, nominal groups were superior, but later, real groups did better. However, it is noteworthy that during the last $5 \mathrm{~min}$, both real and nominal groups produced an average of about half a word, in contrast to the production of approximately three to six words per minute at the start of the time period. Apparently, the pool of available words in the language or in the functional vocabulary of the Ss was nearly exhausted.

It is possible that this apparent ceiling on performance prevented nominal groups from maintaining their pace of output throughout the time period. Had more words been possible,

* Requests for reprints should be sent to Milton E. Rosenbaum, Dept. of Psychology, University of Iowa, Iowa City, Iowa 52240. the superiority of nominal groups may have been maintained. Alternately, it is also possible that, given much more time and more solution possibilities, real groups might exceed nominal groups in productivity. In the present experiment, these alternatives are examined by extending both the task and the time available for problem solving.

\section{DESIGN}

In a 2 by 3 experimental design, one variable was type of group, real vs nominal. The other variable was time available for the anagrams task, the three levels of which were 15,50 , and $105 \mathrm{~min}$.

\section{SUBJECTS}

The Ss were 120 female students from introductory courses in psychology at the University of Iowa, who participated in the experiment to fulfill course requirements. The real groups were made up of 4 Ss working together as a team on the anagram task. There were 5 real groups at each time level, the total number of real groups being 15 and the total number of Ss in the real groups being 60 . Each of the remaining $60 \mathrm{Ss}$ worked alone on the anagrams task, with 20 Ss per each time level. At each time level, 5 nominal groups were formed by pooling the performance of 4 individual Ss. This nominal grouping was based on the order of appearance at the laboratory, Ss 1-4 comprising Nominal Group 1 , Ss $5-8$ comprising Nominal Group 2, and so on. \section{PROCEDURE}

The Ss, either alone or in a group of four, were seated at a large table facing a blackboard. They were told that they would be given a set of letters and that they were to construct as many words as they could out of the letters in the given set, with the restriction that one-letter words, words using a letter more than once, proper names, foreign words, and abbreviations were not acceptable. response potentialities in the anagrams
When a S came up with a word, she was to speak it out and then spell the same, so that $E$ could write it down on his record sheet. Ss were given pencils and scratch paper to make use of if they felt inclined to do so. In the case of real groups, Ss were told that they were working on a group enterprise, each word being scored for the group, irrespective of who gave it. The group members were advised to avoid duplication of words given by other members and were allowed to discuss and consult with one another on any matter such as spelling or admissibility of a particular word.

After the instructions were given, Ss worked on a practice task for $3 \mathrm{~min}$. The letters for the practice task were "T R E A," the same as the ones used by Anderson (1961) for his practice task. The letters were printed on the blackboard by $E$. After the practice period was over, E erased the practice set and printed a set of 12 letters for the experiment proper. The letters in this set were "A F E L G I Y O M B P $T$." Anderson (1961) had used the letters " $A$ F $L$ I $Y$ B $T$ " for his experimental session. The present study used Anderson's eight letters and four additional letters to make up the experimental set.

The available time was announced to Ss before the beginning of the experimental session. The $E$ recorded all the words given by Ss, marking off 5 -min periods with the help of a stopwatch.

The procedure of the present study was essentially similar to that of Anderson (1961). In all cases, scoring was based on the total number of admissible words given once. The score of a nominal group, for example, was obtained by adding the number of admissible words given by each of the four individuals after rejecting all duplicates.

RESULTS
Nominal groups made more words than real groups at each of the three time levels. For both real and nominal groups, performance increased directly as a function of available time. The mean words produced by real and nominal groups are presented in Table 1 , along with the mean words produced by individuals working alone. In an analysis of variance for words made by real and nominal groups, the main effect of group type was significant $(\mathrm{F}=23.16$, $\mathrm{df}=1 / 24$, $\mathrm{p}<.001$ ), and so was the main effect of available time $(F=92.03, \mathrm{df}=$ $2 / 24, p<.001)$. There was no interaction between group type and available time $(F<1.00)$.

\section{DISCUSSION}

The results are clear in demonstrating that extending time for problem solving when solution 
Table 1

Mean Words Made by Individuals, Real Groups, and Nominal Groups

\begin{tabular}{lrrr}
\hline & & Time & \\
\cline { 2 - 4 } & $15 \mathrm{Min}$ & $50 \mathrm{Min}$ & $105 \mathrm{Min}$ \\
\hline Individuals & $\mathbf{7 2 . 1 0}$ & 121.50 & 158.35 \\
Real Groups & 126.00 & 194.20 & 232.20 \\
Nominal Groups & 157.60 & 225.20 & $\mathbf{2 6 2 . 6 0}$ \\
\hline
\end{tabular}

Note-There were 20 individuals, 5 real groups, and 5 nominal groups at each time level.

potentialities are not limited does not yield equivalent performance from real and nominal groups. Nominal groups produced more words than real groups at all levels of available time. Anderson's (1961) findings seem to be based on the inability of the individuals who comprised the nominal groups to continue to produce words. Only when the repertories of the nominal groups were nearly exhausted were the real groups able to catch up in the remaining time.

During the first $15 \mathrm{~min}$ of the present experiment, real and nominal groups were producing words at an average rate of between approximately 8 and 10 words per minute, and, even when the time available was $105 \mathrm{~min}$, the groups produced about 2.5 words on the average during the last $5 \mathrm{~min}$. It appears that the present $\mathrm{Ss}$, even after much passage of time, were capable of producing words at a rate far above that of Anderson's Ss. In fact, the total number of acceptable words that were catalogued from the protocols in the present experiment was 432 . The largest total produced by any single group, real or nominal, in this experiment was 278 in $105 \mathrm{~min}$, which suggests that a ceiling for performance had not been reached even at the highest level of available time.

The results of the present study and those of Watson are consistent with the results of two brainstorming studies (Taylor, Berry, \& Block, 1958; Dunnette, Campbell, \& Jaastad, 1963) in which nominal groups were found to be more productive than real groups. However, in a partial replication of the Taylor et al (1958) study, in which $12 \mathrm{~min}$ had been allowed, Panman \& Rosenbaum (1963) found that when more than four times as much time was given, the productivity of nominal and real groups was not significantly different. At the end of $50 \mathrm{~min}$, both real and nominal groups were producing solutions at a high rate, suggesting that a ceiling effect was not responsible for the similar productivity. It remains possible that had Panman and Rosenbaum's Ss been allowed as much as $105 \mathrm{~min}$, real groups might have exceeded nominal groups.

The issue here, susceptible to empirical test, appears to be the possibility of differences in multiple-solution tasks that may foster differences in problem-solving processes in groups. The anagrams task, although allowing varying numbers of possible solutions, depending on the specific letters employed in the problem, inherently possesses a definite limit on solution possibilities. At the extreme, 26 letters determine the specific limit of the number of words that are at all possible. In addition, it is likely that interindividual stimulation of solutions is relatively low. The functional vocabularies of participants can be expected to involve extensive overlap, but the more esoteric items that may be generatively available to one participant may, at best, appear in the vocabularies of others at the recognition level.

The tasks employed in brainstorming studies have been open-ended, with possibly infinite solution potentialities. A typical problem deals with the advantages and disadvantages of an additional thumb on each human hand. Such problems are likely to provide wide opportunity for interindividual stimulation. This will be particularly true if mutual criticism of quality is eliminated by the usual brainstorming instructions. Although some overlap in solution potentialities is likely, individual repertoires can be expected to differ extensively. An individual working alone should, at some point, reach a ceiling of response potentiality, and, unless new sources of stimulation are provided, the individual's repertory will appear to be functionally exhausted. Exposure to the responses of another person, a situation which exists in real groups, may provide such new sources of stimulation. Panman \& Rosenbaum (1963) observed that, toward the end of $50 \mathrm{~min}$, the individuals who comprised their nominal groups were silent for increasingly lengthy periods of time, while real groups continued to contribute solutions at a rapid rate. Further study of the contribution of extended periods of time for problem solving by groups in relation to task differences is clearly indicated, but as Kelley \& Thibaut (1969) have strongly suggested, this research should pay attention to the details of the process of group problem solving.

\section{REFERENCES}

ANDERSON, N. H. Group performance in an anagram task. Journal of Social Psychology, 1961, 55, 67-75.

DUNNETTE, M. D., CAMPBELL, J., \& JAASTAD, $K$. The effect of group participation on brainstorming effectiveness for two industrial samples. Journal of Applied Psychology, 1963, 47. 30-37.

KELLEY, H. H., \& THIBAUT, J. W. Group problem solving. In $G$. Lindzey and $E$. Aronson (Eds.). The handbook of social psychology. Vol. 4. (2nd ed.) Reading, Mass: Addison-Wesley, 1969 Pp, 1-101.

PANMAN, R. A., \& ROSENBAUM, M. E. A comparison of real groups and nominal groups in the solution of open-ended problems. Paper presented at the meeting of the Midwestern Psychological Association, St. Louis, May 1964.

SHAW, M. E. Group dynamics: The psychology of small group behavior. New York: McGraw-Hill, 1971.

TAYLOR, D. W., BERRY, P. C., \& BLOCK, C. H. Does group participation when using brainstorming facilitate or inhibit creative thinking? Administrative Science Quarterly, 1958, 3, 23-47.

WATSON, G. B. Do groups think more efficiently than individuals? Journal of Abnormal \& Social Psy chology, 1928, 23, 328-336. 